Article

\title{
Evaluation of Isokinetic Single-Leg Cycling as a Rehabilitation Exercise Following Anterior Cruciate Ligament Reconstruction Surgery
}

\author{
Randolph Hutchison ${ }^{1, *}$ (D) , Jessica Myers ${ }^{2}$, Nicholas Hayden ${ }^{1}$, Lee Shearer ${ }^{1}$, Kaitlin Bruneau ${ }^{1}$ \\ and John D. DesJardins ${ }^{2}$ \\ 1 Department of Health Sciences, Furman University, Greenville, SC 29613, USA; \\ nicholas.hayden@furman.edu (N.H.); lee.shearer@furman.edu (L.S.); knb_011@yahoo.com (K.B.) \\ 2 Department of Bioengineering, Clemson University, Clemson, SC 29613, USA; \\ jemyers@g.clemson.edu (J.M.); jdesjar@clemson.edu (J.D.D.) \\ * Correspondence: randolph.hutchison@furman.edu; Tel.: +864-294-3687; Fax: +864-294-2942
}

Received: 26 July 2017; Accepted: 21 August 2017; Published: 31 August 2017

\begin{abstract}
The anterior cruciate ligament (ACL) is one of the most commonly injured ligaments, with over 250,000 injuries per year in the United States. Previous studies have found that ACL-deficient individuals avoid use of the quadriceps in the injured limb as a means of limiting anterior movement of the tibia in the absence of a functioning ACL. From these results, a study was designed to investigate the effectiveness of isokinetic single-leg cycling in increasing quadriceps muscle recruitment and activation. Ten control and seven ACL-reconstructed subjects completed a series of $15 \mathrm{~s}$ cycling trials in isokinetic mode at $75 \mathrm{rpm}$, while kinematic, kinetic, and electromyographic data of the lower limbs were collected, with the trials including both double-leg and single-leg cycling. It was hypothesized that there would be an increase in quadriceps muscle activity, peak knee extensor moment, and knee joint power in single-leg cycling when compared to double-leg cycling. The results of the study suggest that single-leg cycling may be an effective exercise in increasing the strength of the quadriceps following anterior cruciate ligament reconstruction surgery. Although no significant changes occurred, the results indicate that, given a specific limb power, more muscle force will be generated from the quadriceps muscle group in single-leg cycling than double-leg cycling.
\end{abstract}

Keywords: quadriceps avoidance; ACL rehabilitation; electromyography; joint kinetics; joint kinematics

\section{Introduction}

The anterior cruciate ligament (ACL) is one of the most commonly injured ligaments in the human body. Currently, there are approximately 250,000 injuries each year in the United States [1]. The rate of incidence of ACL injury varies between sports and between genders, with the highest rates of injury occurring in collegiate soccer, collegiate basketball, and recreational skiing, and the majority of injuries occurring in women [2]. Following injury, athletes must undergo reconstructive surgery to replace the torn ligament. To regain normal motions and strength in the injured leg, they must then go through a rehabilitation program under the guidance of a physical therapist. However, literature has shown that these programs are not always successful in achieving full recovery [3-8]. The results of these studies overwhelmingly show that patients who undergo ACL reconstruction may not attain normal function in the injured limb after a rehabilitation program. One major concern in the rehabilitation program is bringing the quadriceps back to full strength. Weak quadriceps muscles can affect gait of the patient, inhibiting their progression during recovery. Research must focus on determining why patients cannot achieve full strength and how therapy can address this in the future. 
ACL injuries affect more than 120,000 athletes in the US every year, and only two-thirds of those that undergo reconstruction are able to return to sports within a year [9-11]. It is estimated that $25 \%$ of those that do return will injure their knee a second time [12-14]. Patients may practice quadriceps avoidance in the early period of physical therapy if this compensatory mechanism was developed in the period of time between injury and surgery. If this avoidance persists throughout the rehabilitation program during cycling exercises, patients may never return to full function. Therefore, it is imperative that exercises in physical therapy following surgery succeed in strengthening the quadriceps in order to restore normal gait which, in turn, is expected to help prevent re-injury.

A recent study by Hunt et al. investigated the presence of quadriceps avoidance in ACL-deficient individuals during stationary, isokinetic cycling. It was hypothesized that the injured limbs would exhibit reduced net knee joint extensor moments and reduced quadriceps muscle activity, which was supported by the data, but the results did not entirely conclude that this was due to avoidance of the quadriceps muscle group. They reported that subjects were practicing a type of limb attenuation by outputting more work from the uninvolved limb to maintain the necessary cadence [15].

Hunt et al. had hypothesized that cycling may be a good alternative model to study quadriceps avoidance, since cycling and walking have some similarities; they both require each limb to alternate movements of propulsion, and they both have comparable patterns of muscle activation and joint kinematics [16-18]. However, the study by Berchuck et al. reported that quadriceps avoidance was only prevalent in walking, and not in jogging or stair climbing, where knee flexion angles are greater $[15,19]$. Knee flexion angles in cycling typically reach $60^{\circ}$ or more, similar to stair climbing, which would indicate that cycling may not be an exercise that would even cause quadriceps avoidance.

Isokinetic stationary cycling is a common exercise in ACL rehabilitation [20]. Cycling is a closed kinetic chain exercise and is intended to help restore normal motion of the knee and improve range of motion. Isokinetic exercise is specifically defined as the shortening in length of a muscle or muscle group when it contracts at constant speed. To truly experience isokinetic exercise, opposing forces need to be able to quickly change resistance in order for the muscle to change its length at a constant speed throughout a muscle contraction. Some stationary bicycles that have programmable settings for isokinetic exercises can accomplish this type of activity. Previous studies have advocated the use of isokinetic exercises in ACL rehabilitation for their effectiveness in functional performance and for their safety. The force that opposes movement in concentric contractions, theoretically, should not exceed the force generated by the limb [21]. For these reasons, and the possibility that the knee flexion angles in cycling may not allow for quadriceps avoidance, the present study investigates the effectiveness of an isokinetic single-leg cycling exercise in increasing quadriceps activation and use.

The purpose of this study was to determine if single-leg cycling would be an effective rehabilitation exercise after ACL reconstruction surgery in terms of increasing the muscle activation of the quadriceps. This was done by examining the biomechanical changes that occur between doubleand single-leg cycling and determining if these changes indicate that single-leg cycling increases recruitment of the quadriceps, thereby effectively activating those muscles. We hypothesize that single-leg cycling will prompt a greater use of the quadriceps when compared to double-leg cycling. Specifically, we expect to see increased quadriceps activity, increased knee joint power, and an increased knee extensor moment.

\section{Materials and Methods}

Integrated electromyography of three quadriceps muscles and two hamstring muscles were collected over the entire rotation of the pedal to examine the changes in muscle activity in single-leg cycling. Peak ankle, knee, and hip extensor and flexor moments, peak ankle, knee, and hip power, and linear impulse of the pedals during the downstroke were collected, as well. Due to the fact that the quadriceps muscle group is the active muscle group during the downstroke phase, kinematic, and kinetic data collection were focused on that phase of the cycle. 


\subsection{Subjects}

Seven ACL-reconstructed (ACLR) subjects (five males, two females) participated in this study. Subject information, including age, height, and body mass can be found in Table 1 . These subjects were tested between 10 and 22 weeks post-surgery and all of the subjects had full knee range of motion of the injured limb. All subjects and their physical therapists signed an Institutional Review Board (IRB) approved informed consent form prior to testing and were made aware of all risks and discomforts associated with the testing. Five ACLR subjects had received a bone-patellar tendon-bone (BPTB) graft, while the remaining two had received a hamstring tendon graft. This was a revision ACL surgery for one subject (female, age $=22$, height $=172.7 \mathrm{~cm}$, mass $=60.3 \mathrm{~kg}$ ) due to failure of the previous hamstring tendon graft that was put in place five years prior. The replacement for the more recent surgery was a BPTB graft.

Ten control subjects (five males, five females) participated in the study, as well. All control subjects signed an informed consent form acknowledging they had no history of cardiovascular problems or previous lower limb injury. No statistically significant differences in age $(p>0.05)$, height $(p>0.05)$, or body mass $(p>0.05)$ were found between the control group and ACL group.

Subjects were mostly recruited through word of mouth. Flyers were also posted in physical therapy centers in surrounding towns and around Clemson University campus. In order to create an incentive, a modification was made to this IRB-approved study to allow for compensation to be given in the form of $\$ 50$ Amazon gift cards. These were given to the last four ACL subjects tested.

This study only included subjects from the ACLR population that were within 22 weeks post-surgery in order to study a representative population of patients who are in physical therapy following ACL reconstruction surgery. This excludes ACL-deficient individuals who have not undergone reconstruction surgery to replace the ligament. The protocol was designed to study the possible effectiveness of a single-leg cycling rehabilitation exercise. The kinematic, kinetic, and electromyographic (EMG) variables were selected for analysis based off of previous literature review of quadriceps avoidance after ACLR and cycling studies with ACLR populations.

Trials were excluded from analysis if subjects moved to an unseated position on the bike or removed their hands from the handlebars. Data was excluded if subjects stopped cycling before the $15 \mathrm{~s}$ trial was completed, or if any hardware malfunctioned during testing.

Table 1. Mean values for subject information of the control group and Anterior Cruciate Ligament (ACL) group. Mean (standard error).

\begin{tabular}{cccc}
\hline Group & Age (years) & Height $(\mathbf{c m})$ & Body Mass (kg) \\
\hline Control $(n=10)$ & $21.3(1.6)$ & $171.0(8.9)$ & $69.7(9.2)$ \\
ACL $(n=7)$ & $22.1(3.2)$ & $174.2(8.8)$ & $70.8(10.9)$ \\
\hline
\end{tabular}

\subsection{Protocol}

The protocol for this study was previously approved by the University Institutional Review Board. Subjects performed a randomized series of 15-second cycling trials on a stationary lode ergometer in isokinetic mode at $75 \mathrm{rpm}$. A total of 18 trials were completed for each subject, including six with double-leg cycling, six with right-leg cycling, and six with left-leg cycling. Real-time biofeedback of quadriceps muscle activity was shown to the subjects for half of these trials (three double-leg, three right-leg, and three left-leg trials). Each trial began from a complete stop $(t=0 \mathrm{~s})$, and the subjects cycled until the researchers told them to stop $(t=15 \mathrm{~s})$. Subjects were instructed to cycle at the maximum output they were able to produce, and researchers collecting data at the time of testing gave encouraging words to the subject throughout the duration of the trial. Researchers gave a $10 \mathrm{~s}$ warning and a $5 \mathrm{~s}$ warning at 5 and $10 \mathrm{~s}$ into the trial, respectively. A break of 2-3 min was given between trials to prevent fatigue. No subject was unable to complete the 18 trials due to fatigue, pain, or discomfort. 


\subsection{Data Collection, Processing and Analysis}

Subjects performed the cycling trials on an Excalibur Sport Lode Ergometer (Part \#925900, Lode B.V., Groningen, Netherlands) in isokinetic mode set at $75 \mathrm{rpm}$. Seat height was adjusted to the height of the subject's greater trochanter, and distance between the pedal and crankshaft was consistent between pedals and between subjects. Subjects remained seated and kept their hands on the handlebars throughout all trials (Figure 1). The bike clip pedals were each equipped with an ATI force/torque transducer (ATI Industrial Automation, Apex, NC, USA) that collected tri-directional force exerted on the pedals during the trials. The analog signals collected through the force/torque sensors were filtered with a Butterworth low-pass filter with a default frequency cutoff of $6 \mathrm{~Hz}$. These signals were then converted from Volts to Newtons. For the single-leg cycling trials, the opposite pedal was removed from the bike and a counterweight of $97 \mathrm{~N}$ was added to assist in creating momentum for the contralateral pedal during the upstroke phase of single-leg cycling [22,23]. The leg not involved in the trial rested on an elevated step directly behind the bike.

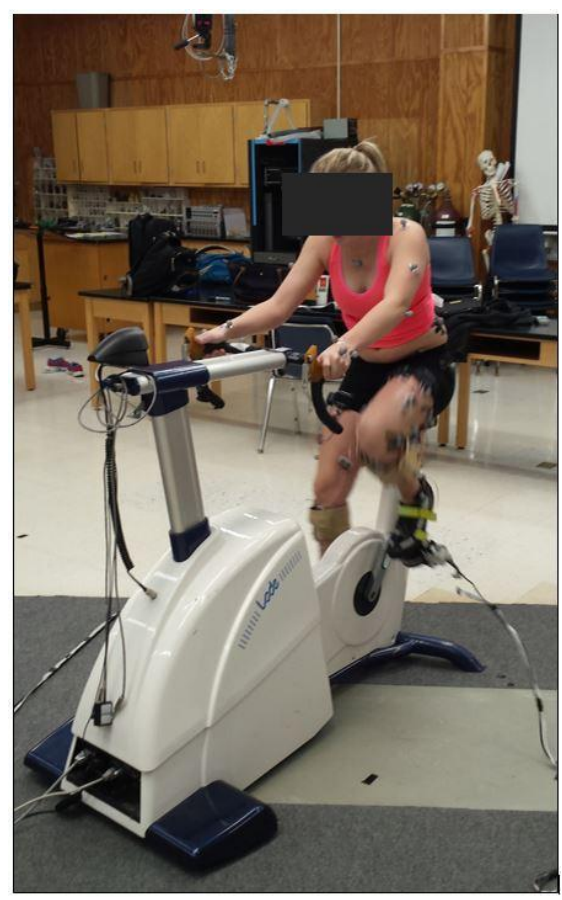

Figure 1. Subject in seated position on ergometer.

Kinematic data was collected through the Pro-Reflex MCU 240 8-camera system (Qualisys AB, Gothenburg, Sweden) at a capture rate of $240 \mathrm{~Hz}$. Kinematic markers were placed on the following locations for each limb: iliac crest, posterior superior iliac spine, greater trochanter, lateral knee joint space, medial knee joint space, lateral malleolus, medial malleolus, proximal heel, distal heel, lateral heel, head of first metatarsal, head of second metatarsal, and head of fifth metatarsal. In addition, four tracking markers were placed on both the lateral thigh and lateral shank. These locations were chosen in accordance with previous marker set guidelines [24], and an example of this setup on the subjects is seen in Figure 2. Four markers were also placed on each pedal, one on its axis, one on its medial side, one on its lateral side, and one on its anterior side, to create pedal coordinate systems. Marker trajectories were labeled using Qualisys Track Manager (Qualisys AB, Gothenburg, Sweden). Motion files were exported to C3D format for inverse dynamics to be performed in Visual 3D (C-Motion Inc., Germantown, MD, USA) to determine joint moments, joint powers, and linear impulses of the pedals. Moments and powers were normalized to subject body mass. Moments were resolved to the proximal coordinate system. For example, the right knee moment was resolved to the right thigh coordinate 
system. Joint power was calculated as the dot product of the joint moment and joint angular velocity. Linear impulses were calculated as an integration of the magnitude of the resultant pedal force in Newtons over the specified time period of the downstroke of the pedal.

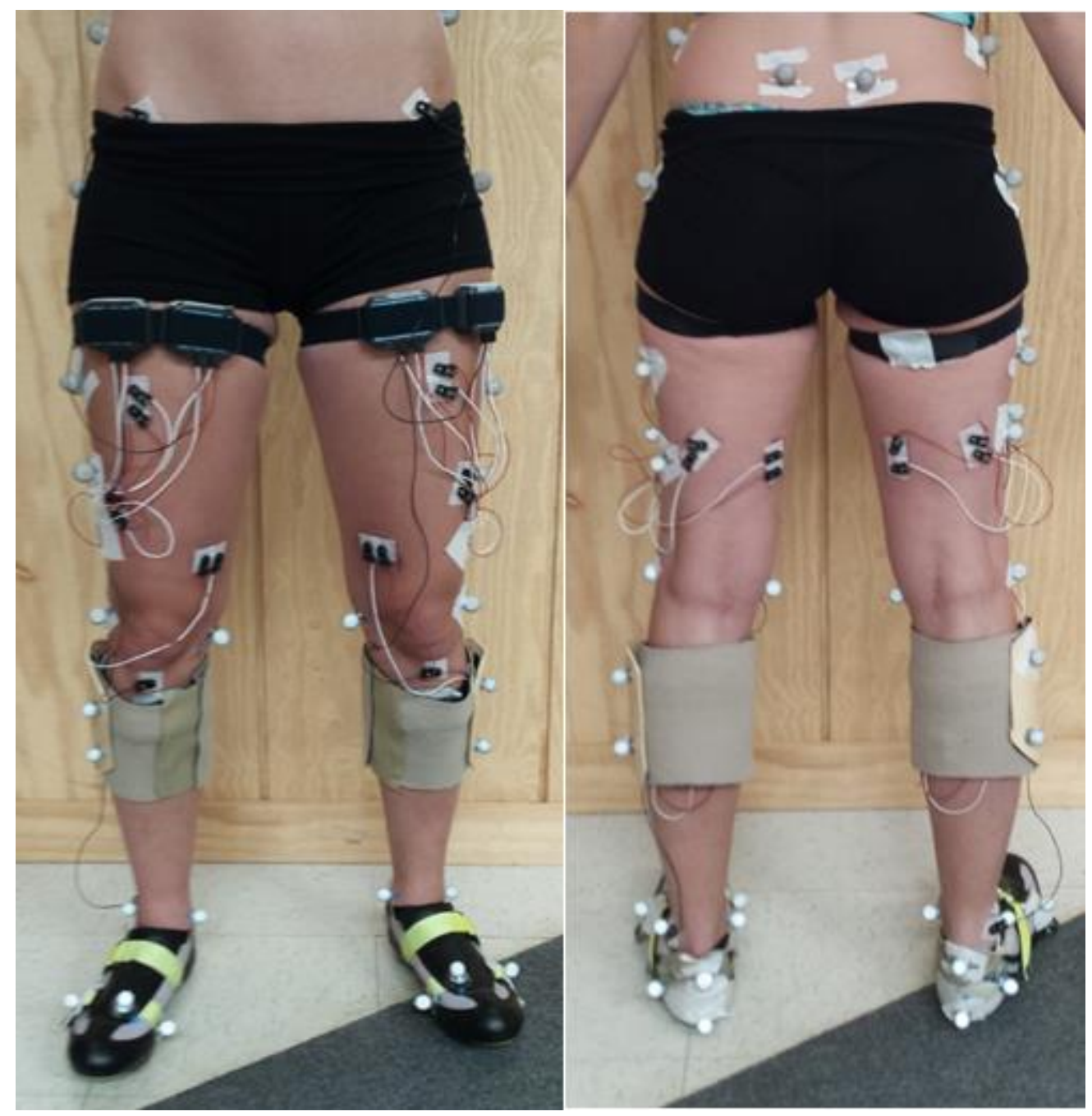

Figure 2. Placement of kinematic markers and electrodes.

Electromyography (EMG) was collected for six muscles on each leg, including the vastus lateralis, vastus medialis, rectus femoris, biceps femoris, semitendinosus, and gastrocnemius. Electrodes were placed on each leg according to the SENIAM model and connected to BioNomadix EMG transmitters (BioPac Systems Inc., Goleta, CA, USA), as seen in Figure 2. Data was collected, processed, and analyzed in AcqKnowledge (Biopac Systems Inc.). A band-pass filter was applied to all data with a high frequency cutoff at $500 \mathrm{~Hz}$, a low frequency cutoff at $10 \mathrm{~Hz}$, and a $\mathrm{Q}$ factor of 0.707 . Data was normalized to the maximum contraction for each muscle found in the testing period. The normalized EMG was integrated for each muscle with a reset period of $0.8 \mathrm{~s}$, which is equivalent to the time of one rotation of the bike at $75 \mathrm{rpm}$.

\subsection{Statistical Analysis}

All statistical tests on data were performed in JMP (SAS Institute Inc., Cary, NC, USA) using a repeated measures analysis of variance. A simple model was used to investigate the effect of leg dominance in the control group, and another simple model was used to look at the effect of biofeedback. A third model was developed to compare the Type (Double or Single) $\times$ Leg (left or right) $\times$ Group 
(Control or ACL) differences in the two subject populations. Specific comparisons were examined between the one limb of the control group and the ACLR limb of the ACL group for ankle, knee, and hip joint powers, ankle, knee, and hip moments, linear impulses of the pedals, quadriceps muscle activity, and hamstrings muscle activity.

\section{Results}

\subsection{Effect of Leg Dominance}

The first analysis was performed on the control group to investigate the effect of leg dominance on all variables of interest. If a large effect exists, there may be a significantly large difference in performance between limbs. The results, outlined in Table 2 , show that leg dominance had a significant effect on the majority of variables of interest $(p<0.01)$. This could be due to better proprioception in the dominant limb, which would allow subjects to cycle with smoother movement of the lower limb, influencing kinematic variables. The dominant leg also outputted significantly more force on the pedals, demonstrating the greater strength of the dominant healthy leg compared to the non-dominant healthy leg. However, this was not supported by the EMG data, which indicated more activity occurred in two of the quadriceps muscles (vastus lateralis and vastus medialis) of the non-dominant leg. Even so, with these results, it can be concluded that it is not possible to compare the ACLR leg of the ACL group to any leg of the control group. Five of the seven subjects tested in the ACL group had surgery in their dominant leg. To account for the two who had surgery in their non-dominant leg $(28 \%$ of the ACL group), we included data of three non-dominant legs of control subjects ( $30 \%$ of the control group) along with data of seven dominant legs of the control subjects to create a comparable control group leg.

Table 2. Effect of leg dominance on variables of interest (standard error values are listed in parentheses).

\begin{tabular}{cccc}
\hline Variable & Non-Dominant Leg & Dominant Leg & $p$-Value \\
\hline Peak ankle extensor moment $(\mathrm{N} \cdot \mathrm{m} / \mathrm{kg})$ & $-0.02(0.12)$ & $-0.04(0.12)$ & 0.3659 \\
Peak ankle flexor moment $(\mathrm{N} \cdot \mathrm{m} / \mathrm{kg})$ & $-0.74(0.15)$ & $-0.85(0.15)$ & $<0.0001$ \\
Peak knee extensor moment $(\mathrm{N} \cdot \mathrm{m} / \mathrm{kg})$ & $1.38(0.26)$ & $1.95(0.26)$ & $<0.0001$ \\
Peak knee flexor moment $(\mathrm{N} \cdot \mathrm{m} / \mathrm{kg})$ & $-1.85(0.23)$ & $-2.14(0.23)$ & 0.0014 \\
Peak hip extensor moment $(\mathrm{N} \cdot \mathrm{m} / \mathrm{kg})$ & $4.51(0.60)$ & $5.74(0.60)$ & $<0.0001$ \\
Peak hip flexor moment $(\mathrm{N} \cdot \mathrm{m} / \mathrm{kg})$ & $-6.10(0.66)$ & $-7.34(0.66)$ & $<0.0001$ \\
Peak ankle power $(\mathrm{W} / \mathrm{kg})$ & $3.28(0.70)$ & $3.73(0.70)$ & 0.0036 \\
Peak knee power $(\mathrm{W} / \mathrm{kg})$ & $8.43(1.33)$ & $12.17(1.33)$ & $<0.0001$ \\
Peak hip power $(\mathrm{W} / \mathrm{kg})$ & $21.86(3.16)$ & $26.42(3.16)$ & $<0.0001$ \\
Linear impulse $(\mathrm{N} \cdot \mathrm{s})$ & $155.6(16.7)$ & $167.1(16.7)$ & 0.0014 \\
Biceps femoris activity $(\mathrm{mV} / \mathrm{s})$ & $4.17(0.29)$ & $4.24(0.29)$ & 0.5792 \\
Rectus femoris activity $(\mathrm{mV} / \mathrm{s})$ & $3.87(0.37)$ & $4.03(0.37)$ & 0.2507 \\
Vastus lateralis activity $(\mathrm{mV} / \mathrm{s})$ & $5.01(0.15)$ & $4.60(0.15)$ & 0.0026 \\
Vastus medialis activity $(\mathrm{mV} / \mathrm{s})$ & $5.85(0.27)$ & $5.02(0.28)$ & $<0.0001$ \\
Semitendinosus activity $(\mathrm{mV} / \mathrm{s})$ & $5.34(0.26)$ & $5.35(0.26)$ & 0.9641 \\
\hline
\end{tabular}

\subsection{Muscle Activity of Quadriceps and Hamstrings}

Integrated EMG values were obtained for quadriceps and hamstrings muscles for both the control and ACL groups in double and single leg cycling, as seen in Figure 3. When looking at the changes that occur in the control limb when switching to single-leg cycling from double-leg cycling, there is a significant decrease in activity in the quadriceps muscles and a significant increase in the hamstrings muscles. The upstroke in cycling is accomplished with force generation from the hamstrings that induces knee flexion and brings the pedal to top dead center. The force required to complete this movement would be much larger without a counter limb to assist in the upstroke, and the counterweight that was implemented in this protocol may not have been successful in mimicking that counter force, which would explain the drastic increase in hamstrings muscle activity for the healthy 
limb. When looking at these changes within the ACL group, however, there is not much of a difference in muscle activity for either the quadriceps or hamstrings. This indicates that the muscle groups are generating the same force in both conditions to accomplish the tasks, as opposed to the control, which generated more muscle force from the hamstrings to accomplish the single-leg cycling task.

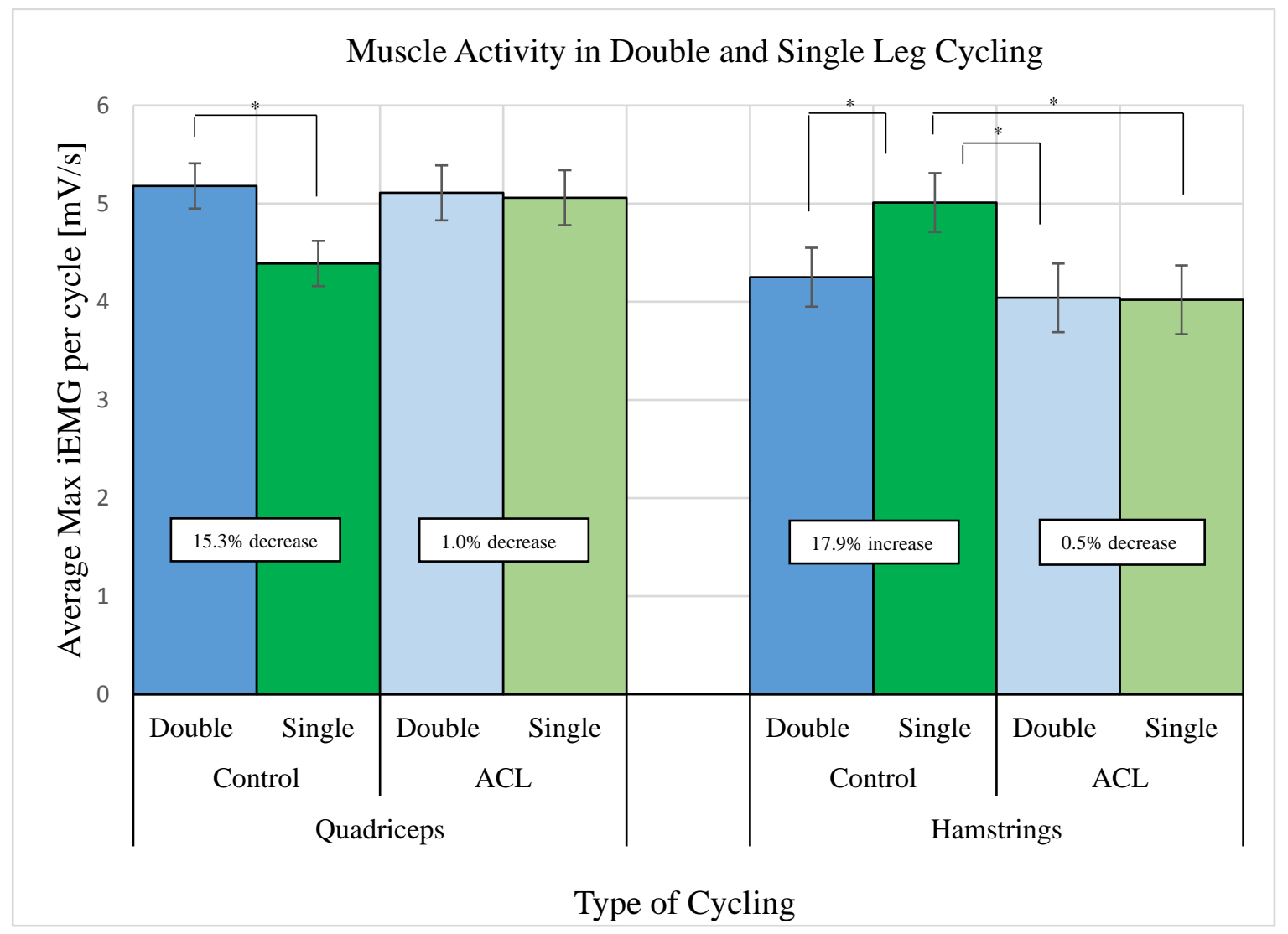

Figure 3. Average integrated EMG (significance $(p<0.05)$ is noted with an asterisk).

\subsection{Joint Powers, Joint Moments, and Pedal Impulses}

Joint power is an indicator of the amount of energy each joint is generating to complete the cycling task in each condition. Figure 4 outlines the results of peak joint power data collected in the downstroke for the ankle, knee, and hip, as well as total peak joint power in this phase. There is no significant difference in power for any joint between double and single leg cycling, but some obvious trends exist. In the control group, there is a decrease in ankle power and in hip power, as well as an increase in knee joint power in single leg cycling compared to double leg cycling. However, in the ACL group, there is a decrease in power in all joints. There is a noticeable difference in the magnitude of hip power between the control limb and ACLR limb, with the ACLR hip generating more power in both double- and single-leg cycling. Looking at total joint power of these limbs, Figure 4 illustrates the decrease in ACLR limb power is almost twice that of the control limb power in single-leg cycling compared to double-leg cycling. These results point towards the difficulty in generating the same amount of energy when cycling with just one limb than with both limbs.

Joint power is calculated as the product of joint moment and joint angular velocity. To further understand why these trends are present in joint power, it is necessary to see what changes are occurring in joint moments. Since the motion of the knee and hip are both in extension during the downstroke phase, the moments influencing peak joint power here are the peak extensor moments. As seen in Figure 5, the same trends are present in knee and hip extensor moments. The control limb exhibits an increase in peak knee extensor moment in single-leg cycling and a decrease in peak hip 
extensor moment, while the ACLR limb exhibits a decrease in both variables. The magnitudes of the hip moments are also much larger in the ACLR limb than the control limb.

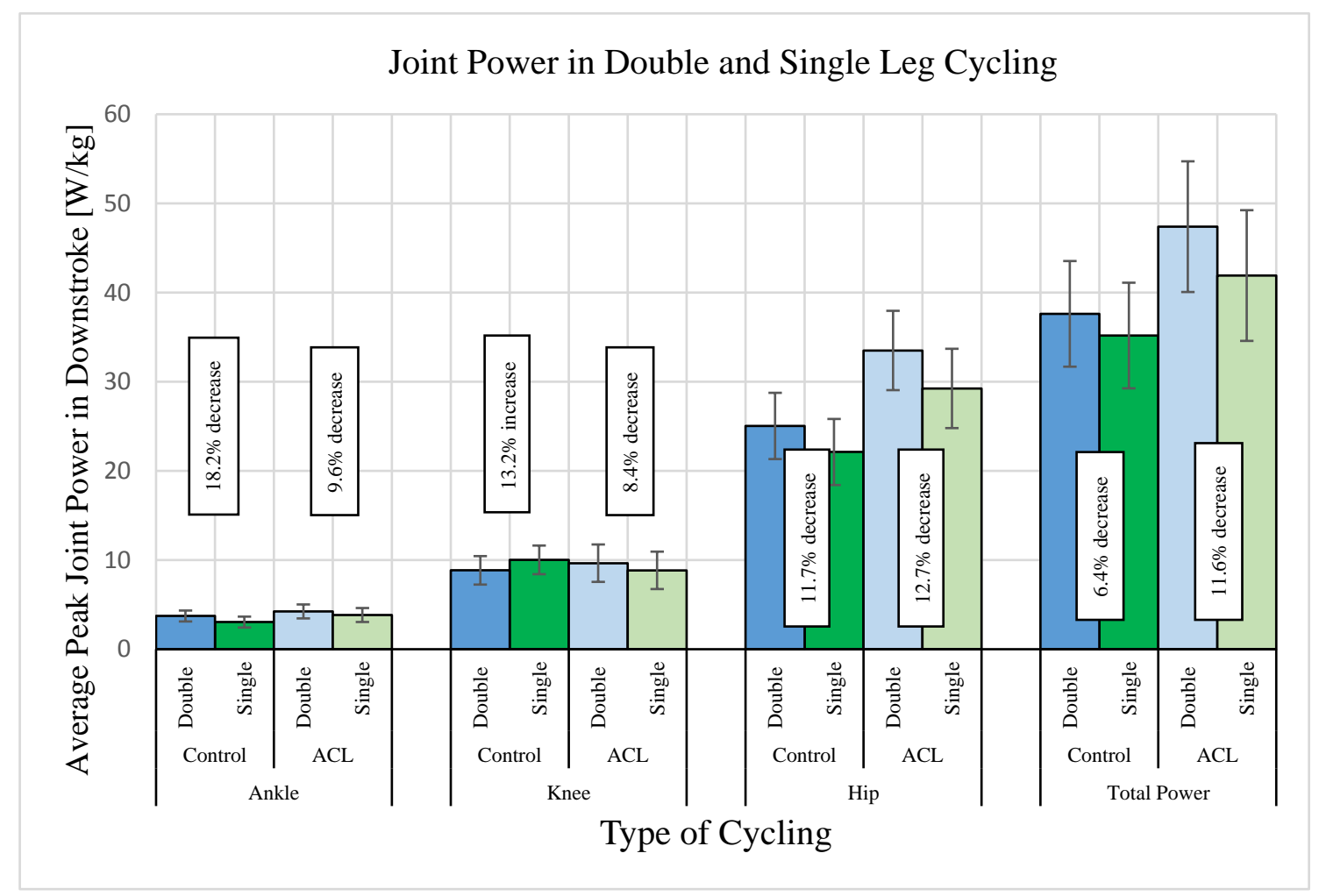

Figure 4. Average maximum joint power in the downstroke (no significance was found; $p<0.05$ ).

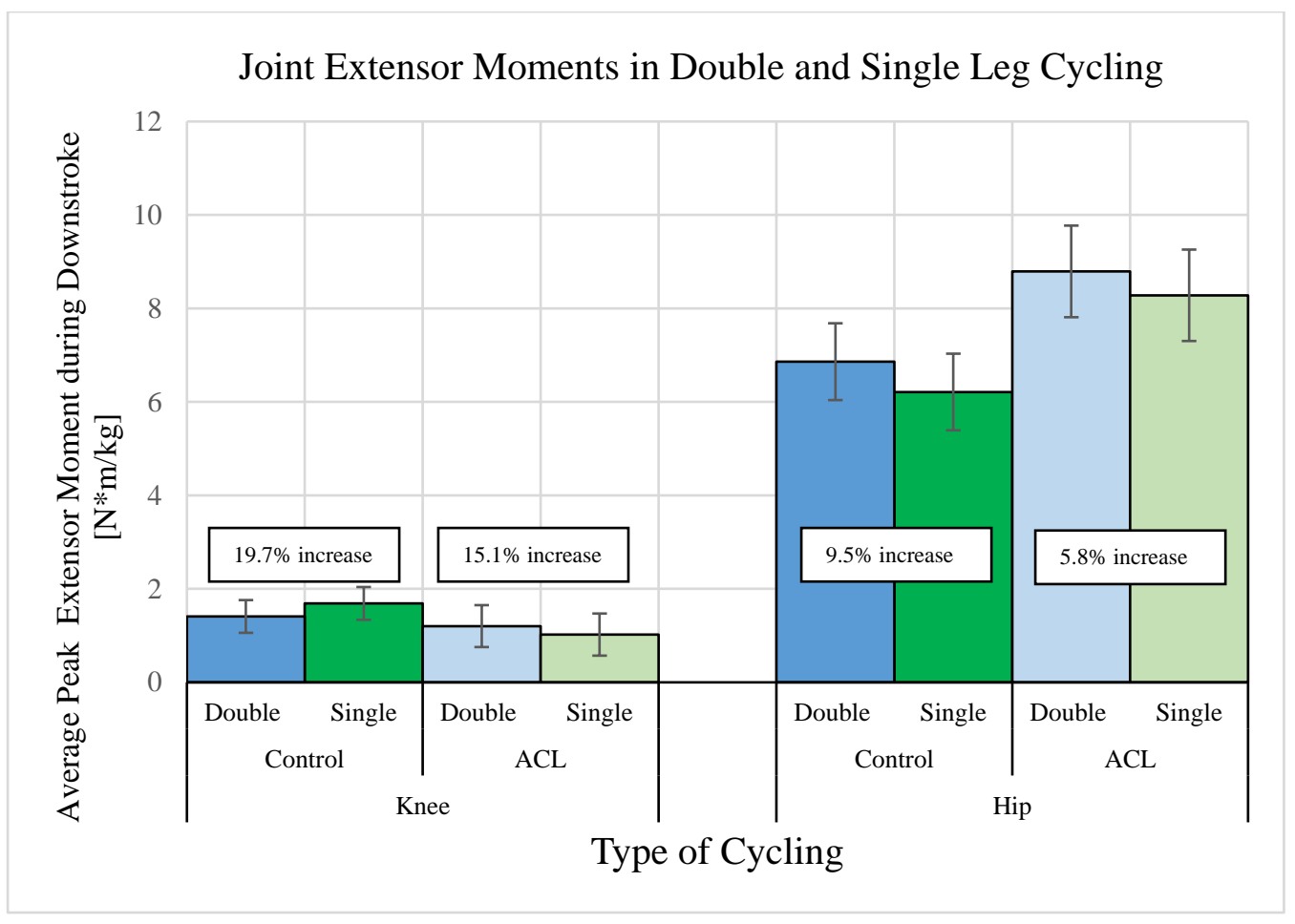

Figure 5. Average peak joint extensor moments in the downstroke (no significance was found; $p<0.05$ ). 
Linear impulses of the pedals were calculated for the downstroke phase. The results are outlined in Table 3. For both the control limb and ACLR limb, there is a reduction in linear impulse in the single-leg task compared to the double-leg task, with slightly higher impulses generated from the ACLR limb compared to the control limb. Since the resultant pedal force is influenced by the amount of power generated by the corresponding limb, these results support the results of the joint power data.

Table 3. Average linear impulse in the downstroke (no significance was found; $p<0.05$ ).

\begin{tabular}{ccc}
\hline \multicolumn{3}{c}{ Linear Impulse in Downstroke [N·s] } \\
\hline & Double Leg Cycling & Single Leg Cycling \\
Control Group & $161.9(17)$ & $152.4(17)$ \\
ACL Group & $177.2(19)$ & $165.8(19)$ \\
\hline
\end{tabular}

\section{Discussion}

Quadriceps avoidance in the gait of ACL-deficient individuals has consistently been defined in previous literature by a reduced knee extensor moment. Joint moments are influenced by two factors: the joint segments' mass moment of inertia and the joint's angular acceleration. The mass moment of inertia is directly related to muscle forces that allow for extension or flexion of a joint. As the mass of a joint segment increases, the force produced by muscles to flex or extend the joint increases as well. The quadriceps muscle group generates a force to induce a knee extensor moment, while the hamstrings group generates a force to induce a knee flexor moment. These past studies on quadriceps avoidance have reported a reduced knee extensor moment, implying a reduction in force generated by the quadriceps, hence the term "quadriceps avoidance". Hunt et al. found that this reduced moment also existed in cycling for ACL-deficient subjects. However, their findings indicated that these subjects almost entirely avoided use of ACL-deficient limbs and the counter limb supplied more energy and power to complete the cycling tasks. The present study investigates the ability of single-leg cycling to promote a greater knee extensor moment, in turn increasing activation of the quadriceps, and thereby exercising that muscle group and increasing its strength.

It was hypothesized that single-leg cycling would cause greater quadriceps muscle activity. Results from this study show that single-leg cycling actually decreased the amount of activity from all three quadriceps in the control group, and did not significantly change the activity of the quadriceps in the ACL group. It appears that the hamstrings of the control limb produced more force in the single-leg task to make up for the large decrease in quadriceps force. These changes are not well explained by the results of the kinematic and kinetic data. With a reduction in quadriceps activity in the single-leg task, a reduction in knee extensor moment would be expected, but the results indicate that there is a slightly larger knee extensor moment. To better understand the kinematic mechanisms associated with this trend, it would be best to investigate the activity of these muscles during the downstroke and upstroke separately. However, due to limitations of the study, this type of analysis was not possible. The control limb may be experiencing co-contraction of the quadriceps and hamstrings during one complete rotation on the bike that results in a greater net activity from the hamstrings as opposed to the quadriceps. For this study, it was assumed that the quadriceps are the major contributor of muscle force for the downstroke and the hamstrings are the major contributor of muscle force for the upstroke. Suggestions for future studies include investigating the activation patterns of the quadriceps and hamstrings during the two phases of single-leg cycling to determine if this assumption is true.

The ACLR limb provided the same amount of muscle activity in both the quadriceps and hamstrings in both types of cycling trials. This indicates that the idea that these muscles were able to output the same amount of force, regardless of changing cycling conditions. Again, this data reflects the activity occurring throughout entire rotations, and does not distinguish activity solely in the downstroke phase. 
It was hypothesized that single-leg cycling would cause an increase in peak knee extensor moment, as well as peak knee joint power. Although not significant, this pattern is present in the healthy control limb, but not present in the ACLR limb. The results also indicate that both control and ACLR limbs do not produce the same amount of total joint power in the single-leg task compared to double-leg task. This is supported by the pedal impulse data, which reports a decrease in total power transferred to the pedals. Although the amount of power of the ACLR limb decreases for the single-leg task, the EMG data proves the quadriceps muscles are still generating the same amount of force. In other words, the ACLR limb is generating more muscle force per any given limb power in single-leg cycling than double-leg cycling.

In addition, the healthy limb shows a slightly larger extensor moment in the knee in the single-leg trials, demonstrating that a single-leg exercise like this, if implemented into a rehabilitation program, may induce more knee extension and more quadriceps force as patients progress through physical therapy and reach a healthy limb status. This increase in joint moment was not exhibited by the ACL group because the testing procedure only assessed the subjects' ability to perform cycling tasks at a certain point in their physical therapy program, and did not take into account the effect of long-term exercise involving single-leg cycling on these variables. Subjects also noted that they were initially uncomfortable with the movements involved in the single-leg trials, and commented on the difficulty in completing them. Future studies should consider testing subjects multiple times throughout physical therapy, and possibly train subjects in single-leg cycling prior to testing in an effort to familiarize them with the motions.

\section{Limitations}

The majority of subjects in this study were generally young and active. Therefore, the results of this study would not be comparable to patients who are older or not active. The time out of surgery when the subjects were tested varied greatly; some subjects were tested in as little as 10 weeks out, and some as far as 22 weeks out. If the study could be redesigned, it is suggested that subjects come in for testing multiple times during their physical therapy program to investigate how their ability to perform single-leg cycling exercises changes throughout the program.

The kinematic and kinetic variables were analyzed in the downstroke $\left(0-180^{\circ}\right)$ and upstroke $\left(180-360^{\circ}\right)$ of the pedal to differentiate the periods of when the quadriceps and hamstrings are responsible for the work done. However, this was not done with EMG data due to an inability to differentiate downstroke and upstroke periods, so analysis was performed for $0.8 \mathrm{~s}$ intervals, which is equivalent to the time to complete one rotation of the bike at $75 \mathrm{rpm}$. It was assumed that subjects cycled at a constant cadence of $75 \mathrm{rpm}$, but the isokinetic mode of the Excalibur Sport lode ergometer has a variability of $+/-6 \mathrm{rpm}$ at this speed.

The protocol was designed to test the performance of subjects over a series of 18 trials. Many variables were investigated in this study, including single-leg cycling versus double-leg cycling and the presence of biofeedback versus no biofeedback, which contributed to the need for a protocol involving many trials. One shortcoming of this is that the trials could only last for $15 \mathrm{~s}$ in order to prevent fatigue of the subjects during testing.

While compensation for ACL-deficient limbs by ACL-intact limbs has been demonstrated at increasing submaximal intensities by Hunt et al., there may be a limit to extending this mechanism to a maximal effort, as in our study. Protocols for future studies may look into exploring increasing intensity levels towards maximum efforts to define where compensation may no longer occur.

\section{Conclusions}

The results of the study suggest that single-leg cycling may be an effective exercise in increasing the strength of the quadriceps following anterior cruciate ligament reconstruction surgery. Although no significant changes occurred, the results indicate that given a specific limb power, more muscle force will be generated from the quadriceps muscle group in single-leg cycling than double-leg cycling. 
Also not significant, the control group exhibited an increased knee flexor moment when changing to single-leg cycling. This reveals a possible increase in quadriceps muscle force in this type of activity.

The sample size of this study was small, which limited the statistical significance of many variables of interest in this study. Subjects in this study were predominantly young and active, so results are not representative of the entire ACL-reconstructed population. Future studies should encompass a wider range of individuals, as well as a larger number of subjects. The ACLR individuals were only tested at one point in their physical therapy program, and the phase in which each individual had reached in the program varied. When investigating the possible effectiveness of single-leg cycling in the future, it would be best to test subjects at multiple time points after ACL reconstruction surgery.

It should also be noted that muscle activation patterns may change in single-leg cycling tasks, and this phenomenon should be further investigated. Results from the EMG data were not easily explained by the kinematic results, due to differences in when data was recorded. It is suggested that future studies focus on data collection in the phase of cycling in which quadriceps muscle activation occurs in order to better understand the relationship between muscle forces, joint moments, and joint powers during single-leg cycling, and what implications it may have for increasing quadriceps strength.

Due to limitations, the protocol for testing involved extremely short sprint cycling tests, which differs from typical rehabilitation exercises involving cycling. In physical therapy, patients will normally use a stationary bicycle to exercise for a period of 15-30 min. More research needs to be conducted to determine what kind of single-leg cycling protocol would be safe, effective, and applicable in a physical therapy setting.

Acknowledgments: There is no funding to report in completion of this research study. We would like to thank Ray Moss of Furman University for his assistance with the motion capture lab facilities. We would also like to thank William Bridges for his assistance with statistical analysis.

Author Contributions: Randolph Hutchison, Jessica Myers, and John D. DesJardins conceived, designed, performed experiments, analyzed data, contributed materials/analysis tools, and wrote the paper. Kaitlin Bruneau, Lee Shearer, and Nicholas Hayden contributed significantly to the formatting and revision of the paper.

Conflicts of Interest: Randolph Hutchison, Jessica Myers, Kaitlin Bruneau, Lee Shearer, and Nicholas Hayden have no potential conflicts to disclose. John D. DesJardins has not received any payments or services from a third party for any aspect of the submitted work; Outside of the submitted work, he reports grants from National Science Foundation, personal fees and other from DJO Surgical, non-financial support from Smith \& Nephew, grants from Arthrosurface, and grants from Hawkins Foundation; In addition, John D. DesJardins has a patent, Lockable Knee Implants and Related Methods, pending.

\section{References}

1. Dai, B.; Herman, D.; Liu, H.; Garrett, W.E.; Yu, B. Prevention of ACL injury, part I: Injury characteristics, risk factors, and loading mechanism. Res. Sports Med. (Print) 2012, 20, 180-197.

2. Prodromos, C.C.; Han, Y.; Rogowski, J.; Joyce, B.; Shi, K. A Meta-analysis of the Incidence of Anterior Cruciate Ligament Tears as a Function of Gender, Sport, and a Knee Injury-Reduction Regimen. Arthroscopy-Arthroscopy 2007, 23, 1320-1325. [CrossRef] [PubMed]

3. Arangio, G.A.; Chen, C.; Kalady, M.; Reed, J.F. Thigh muscle size and strength after anterior cruciate ligament reconstruction and rehabilitation. J. Orthop. Sports Phys. Ther. 1997, 26, 238-243. [CrossRef] [PubMed]

4. Arms, S.W.; Pope, M.H.; Johnson, R.J.; Fischer, R.A.; Arvidsson, I.; Eriksson, E. The biomechanics of anterior cruciate ligament rehabilitation and reconstruction. Am. J. Sports Med. 1984, 12, 8-18. [CrossRef] [PubMed]

5. Keays, S.L.; Bullock-Saxton, J.; Keays, A.C.; Newcombe, P. Muscle strength and function before and after anterior cruciate ligament reconstruction using semitendinosus and gracilis. The Knee 2001, 8, 229-234. [CrossRef]

6. Kowalk, D.L.; Duncan, J.A.; McCue, F.C.; Vaughan, C.L. Anterior cruciate ligament reconstruction and joint dynamics during stair climbing. Med. Sci. Sports Exerc. 1997, 29, 1406-1413. [CrossRef] [PubMed]

7. Paterno, M.V.; Ford, K.R.; Myer, G.D.; Heyl, R.; Hewett, T.E. Limb asymmetries in landing and jumping 2 years following anterior cruciate ligament reconstruction. Clin. J. Sport Med. 2007, 17, 258-262. [CrossRef] [PubMed]

8. Williams, G.N.; Snyder-Mackler, L.; Barrance, P.J.; Axe, M.J.; Buchanan, T.S. Muscle and tendon morphology after reconstruction of the anterior cruciate ligament with autologous semitendinosus-gracilis graft. J. Bone Joint Surg. Am. 2004, 86, 1936-1946. [CrossRef] [PubMed] 
9. Ardern, C.L.; Webster, K.E.; Taylor, N.F.; Feller, J.A. Return to the preinjury level of competitive sport after anterior cruciate ligament reconstruction surgery: Two-thirds of patients have not returned by 12 months after surgery. Am. J. Sports Med. 2011, 39, 538-543. [CrossRef] [PubMed]

10. Frank, C.B.; Jackson, D.W. The science of reconstruction of the anterior cruciate ligament. J. Bone Joint Surg. Am. 1997, 79, 1556-1576. [CrossRef] [PubMed]

11. Majewski, M.; Susanne, H.; Klaus, S. Epidemiology of athletic knee injuries: A 10-year study. The Knee 2006, 13, 184-188. [CrossRef] [PubMed]

12. Hui, C.; Salmon, L.J.; Kok, A.; Maeno, S.; Linklater, J.; Pinczewski, L.A. Fifteen-year outcome of endoscopic anterior cruciate ligament reconstruction with patellar tendon autograft for "isolated" anterior cruciate ligament tear. Am. J. Sports Med. 2011, 39, 89-98. [CrossRef] [PubMed]

13. Leys, T.; Salmon, L.; Waller, A.; Linklater, J.; Pinczewski, L. Clinical results and risk factors for reinjury 15 years after anterior cruciate ligament reconstruction: a prospective study of hamstring and patellar tendon grafts. Am. J. Sports Med. 2012, 40, 595-605. [CrossRef] [PubMed]

14. Shelbourne, K.D.; Gray, T.; Haro, M. Incidence of subsequent injury to either knee within 5 years after anterior cruciate ligament reconstruction with patellar tendon autograft. Am. J. Sports Med. 2009, 37, $246-251$. [CrossRef] [PubMed]

15. Hunt, M.A.; Sanderson, D.J.; Moffet, H.; Inglis, J.T. Biomechanical changes elicited by an anterior cruciate ligament deficiency during steady rate cycling. Clin. Biomech. 2003, 18, 393-400. [CrossRef]

16. Gregor, R.J.; Cavanagh, P.R.; LaFortune, M. Knee flexor moments during propulsion in cycling-a creative solution to Lombard's Paradox. J. Biomech. 1985, 18, 307-316. [CrossRef]

17. Mohr, T.; Allison, J.D.; Patterson, R. Electromyographic Analysis of the Lower Extremity during Pedaling. J. Mot. Behav. 1981, 2, 163-170. [CrossRef] [PubMed]

18. Winter, D.A. Biomechanical motor patterns in normal walking. J. Mot. Behav. 1983, 15, 302-330. [CrossRef] [PubMed]

19. Berchuck, M.; Andriacchi, T.P.; Bach, B.R.; Reider, B. Gait adaptations by patients who have a deficient anterior cruciate ligament. J. Bone Joint Surg. Am. 1990, 72, 871-877. [CrossRef] [PubMed]

20. Van Grinsven, S.; van Cingel, R.E.H.; Holla, C.J.M.; van Loon, C.J.M. Evidence-based rehabilitation following anterior cruciate ligament reconstruction. Knee Surg. Sports Traumatol. Arthrosc. 2010, 18, 1128-1144. [CrossRef] [PubMed]

21. Tsaklis, P.; Abatzides, G. ACL rehabilitation program using a combined isokinetic and isotonic strengthening protocol. Isokinet. Exerc. Sci. 2002, 10, 211-219.

22. Abbiss, C.R.; Karagounis, L.G.; Laursen, P.B.; Peiffer, J.J.; Martin, D.T.; Hawley, J.A.; Fatehee, N.N.; Martin, J.C. Single-leg cycle training is superior to double-leg cycling in improving the oxidative potential and metabolic profile of trained skeletal muscle. J. Appl. Physiol. 2011, 110, 1248-1255. [CrossRef] [PubMed]

23. Thomas, L.N. Single leg cycling: an evaluation of pedal powers. Med. Sci. Sports Exerc. 2009, 41, 54-55. [CrossRef]

24. Selbie, W.; Hamill, J.; Kepple, T. Three-dimensional kinetics. In Research Methods in Biomechanics, 2nd ed.; Human Kinetics: Champaign, IL, USA, 2014; pp. 151-176.

(C) 2017 by the authors. Licensee MDPI, Basel, Switzerland. This article is an open access article distributed under the terms and conditions of the Creative Commons Attribution (CC BY) license (http://creativecommons.org/licenses/by/4.0/). 